\title{
An Unusual Case of Fibroma of the Uterus, removed by Supra-vaginal Hysterectomy.*
}

\author{
By W. K. WaLrs, M.B. (Lond.), \\ Obstetric Assistant Surgeon, St. Mary's Hospital, Manchester.
}

THE following brief particulars-from notes by Dr. Clifford, house surgeon to St. Mary's Hospital, Manchester-of a case of supravaginal hysterectomy for fibroma uteri, appear worthy of record. The case was specially interesting because of the discovery, and continued growth of the tumour some years after an unusually early climacteric in a nulliparous woman; also because of the operation vicissitudes of the patient; and further, inasmuch as a considerable portion of the tumour occupied a large ventral hernia, and had increased in size whilst in that position.

The patient, M.M., aged 44 years, a charwoman, was admitted to St. Mary's Hospital, Manchester, on July 20th, 1904. She had been married 20 years, had never been pregnant, and her last period was nine years before.

She noticed a large lump in the lower abdomen some six years ago. About five years since she states she was operated upon; there was a second operation six weeks later, and a third four weeks after that. She remained in hospital twelve months. From her description, the first operation would appear to have been an abdominal section, whilst the other two were for the relief of abscesses.

During the last five years the tumour had grown considerably, and it had come much more forward. About two months ago the skin over it became ulcerated in patches, with a large amount of purulent discharge. At that time she came under the care of Dr. Higginson, of Bolton, with whom Dr. Walls first saw the case in consultation.

On examination the abdomen was found considerably and irregularly enlarged. At the lower part was a large ventral hernia about the size of a boy's head. The skin over it was brownish, indurated, and scarred. In three places there were ulcerated patches about $3 \times 1 \frac{1}{2}$ inches in size. Filling the hernia was a firm, rounded mass, attached posteriorily to a larger tumour in the abdomen, which gave rise to some prominence on each side of the rupture, and extended from the pubes to the umbillicus. This was dull on percussion and possessed of only a little mobility from side to side.

* Read at a meeting of the North of England Obstetrical and Gynacological Society, October 21, 1904 . 
$P . V$. The vagina was very elongated, and the cervix drawn upwards and only indefinitely to be felt. The lower aspect of the tumour was reached on each side, and the uterus appeared to be involved in the mass. There was a fistula-in-ano.

August 27th, 1904. Operation. The hernial sac was opened by a vertical incision extending its whole length, and the tumour found to consist of multiple fibromata of the uterus. Both broad ligaments seemed involved, the omentum was adherent behind, as was the abdominal wall in front on the left side. The disposition of the fibroids and the almost complete fixation of the whole mass were such that whilst the left tube and ovary were at length made out, the right broad ligament could not be felt at all. An incision was then made into the most prominent part of the tumour, and fourteen fibroids, varying in size from that of a hen's egg to that of an orange, were enucleated, after which the uterus and remaining fibroids were removed by ligaturing the broad ligaments from above downwards, pulling up such of the fibroids as invaded them, and making a stump of the cervix in the usual way, covering it over with peritoneum. The right ovary was found adherent to the antero-lateral pelvic wall, and it was left there, the ligatures being applied between it and the uterus. The left appendages were removed.

$\Delta$ good deal of the hernial sac was taken away, and the abdominaI wound closed in two layers, but owing to the woman being rather collapsed it appeared inadvisable to prolong the operation by performing a radical cure of the hernia, as had been intended. This was unfortunate, as the unhealthy condition of the skin led to some stitches giving way on September 6th, i.e., ten days after operation, and bowel appeared in the wound, necessitating further suturing to cover it. Otherwise the after progress was perfectly satisfactory, and the patient left hospital on October 22nd, 1904.

The uterus and tumours weighed $11 \mathrm{lbs}$. 